\title{
Probing the nuclear structure in the vicinity of ${ }^{78} \mathrm{Ni}$
}

\author{
E. Sahin ${ }^{1, *}, K$. Hadyńska-Klęk $\mathrm{k}^{1,2,3,4}$, F.L. Bello Garrote ${ }^{1}$, and A. Görgen ${ }^{1}$ for the EURICA and SEASTAR collaborations \\ ${ }^{1}$ University of Oslo, Department of Physics, Oslo, Norway. \\ ${ }^{2}$ Laboratori Nazionali di Legnaro dell'INFN, Legnaro, Italy \\ ${ }^{3}$ University of Surrey, Department of Physics, Surrey, UK \\ ${ }^{4}$ Heavy Ion Laboratory, University of Warsaw, Poland
}

\begin{abstract}
Theoretical and experimental studies of neutron-rich nuclei have shown that the general concept of shell structure is not as robust and universal as earlier thought, but can exhibit significant changes as a function of neutron excess. New magic numbers appear and some other conventional ones disappear mainly because of a different ordering of the single-particle orbitals. In the present contribution, recent experimental studies of neutron-rich $\mathrm{Cu}$ isotopes, performed at RIKEN using $\beta$ decay and one-proton knockout reactions, will be discussed. Neutron-rich nuclei near ${ }^{78} \mathrm{Ni}$ were populated through in-flight fission of ${ }^{238} \mathrm{U}$ on thick ${ }^{9} \mathrm{Be}$ targets in both experiments. In the $\beta$-decay study, ${ }^{75,77} \mathrm{Ni}$ nuclei were implanted into the WAS3ABi silicon array, while $\gamma$ rays from excited states in ${ }^{75,77} \mathrm{Cu}$ emitted after $\beta$ decay of the implanted ions were detected with the EURICA Ge detector array that was surrounding the active stopper. In a second experiment within the SEASTAR campaign at RIKEN, the same ${ }^{75,77} \mathrm{Cu}$ nuclei were produced in $(\mathrm{p}, 2 \mathrm{p})$ knockout reactions from ${ }^{76,78} \mathrm{Zn}$ beam particles at around $250 \mathrm{MeV} /$ nucleon impinging onto the MINOS liquid hydrogen target. In the latter experiment the DALI2 NaI array was used to detect de-excitation $\gamma$ rays measured in coincidence with $\mathrm{Cu}$ nuclei identified in the Zero Degree Spectrometer. Both studies are complimentary and greatly contribute to our understanding on the nuclear structure in the ${ }^{78} \mathrm{Ni}$ region.
\end{abstract}

\section{Introduction}

Over the last two decades, investigations at radioactive ion beam facilities have revealed that atomic nuclei undergo significant changes in their shell structure when moving towards neutron-rich isotopes. This so-called shell evolution for nuclei with large neutron-to-proton asymmetry is due to specific properties of the nucleon-nucleon interaction, which can lead to a different ordering of singleparticle orbitals and, hence, to the appearance or disappearance of shell gaps. The phenomenon is by now well established in lighter mass regions with $Z<28$ and $N<40$.

As an example, Figure 1a shows the energies of the first excited $2^{+}$states, $E\left(2_{1}^{+}\right)$, as a function of neutron number $N$ for isotopes of $\mathrm{Si}, \mathrm{S}, \mathrm{Ar}$, and $\mathrm{Ca}$. One can clearly see the disappearance of the neutron $N=28$ shell gap in ${ }^{42} \mathrm{Si} \mathrm{[1],}{ }^{44} \mathrm{~S}$ [2], and ${ }^{46} \mathrm{Ar}$ [3], as evidenced by their low $E\left(2_{1}^{+}\right)$energies. On the other hand, the excitation energies for ${ }^{52,54} \mathrm{Ca}$ are very high, indicating the appearance of new shell gaps at $N=32$ and 34 . The shell gap at $N=34$ appears to be rather robust, as illustrated by the high excitation energy of the $2_{1}^{+}$state in ${ }^{52} \mathrm{Ar}$ [4]. The reduction or even collapse of shell closures when going far away from stability is connected with an onset of deformation. The phenomenon where deformation is favored due to particle-hole excitations across major shells has been

\footnotetext{
*e-mail: eda.sahin@fys.uio.no
}

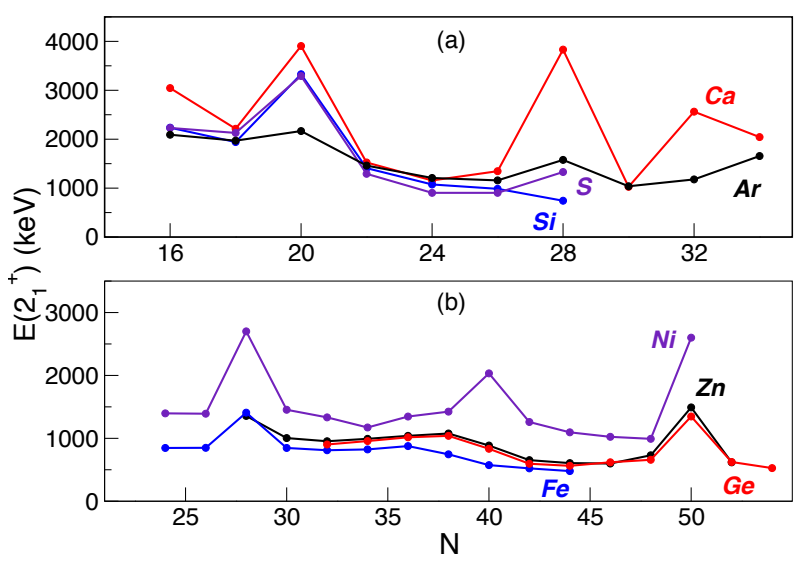

Figure 1. First excited $2^{+}$states as a function of neutron number (a) for $\mathrm{Si}, \mathrm{S}, \mathrm{Ar}, \mathrm{Ca}$ and (b) for Fe, Ni, Zn, and Ge isotopes. Data is taken from References [4, 6, 7].

termed "island of inversion". In general, the coexistence of intruder and normal configurations leads to shape coexistence, which consequently can be expected in regions of disappearing shell closures. As an illustrative example ${ }^{44} \mathrm{~S}$ can be mentioned, where a prolate $0_{1}^{+}$ground state coexists with a spherical excited $0_{2}^{+}$state [5]. In this case the reduction of the $N=28$ shell gap enables quadrupole excitations from the $1 f_{7 / 2}$ to the $2 p_{3 / 2}$ orbitals. 
In the light of these results, questions arise concerning the robustness of the $N=50$ shell gap for neutron-rich nuclei, the magic character of ${ }^{78} \mathrm{Ni}$, and possible intruder states and an onset of deformation in the region around ${ }^{78} \mathrm{Ni}$. Experimental information in this region of the nuclear chart is very sparse due to the extreme proton-toneutron ratio, which makes production of these nuclei difficult and experimental studies in general very challenging. The energy of the $2_{1}^{+}$state in ${ }^{78} \mathrm{Ni}$ was only very recently measured to be $2.6 \mathrm{MeV}$ [6]. This high energy supports the notion of doubly magic character for ${ }^{78} \mathrm{Ni}$ and the persistence of the $Z=28$ and $N=50$ shell gaps (see Figure 1b). However, detailed studies on the evolution of single-particle orbitals in this mass region are still very much incomplete. The aim of the experiments that are presented here was to contribute to a more quantitative understanding of the shell structure near ${ }^{78} \mathrm{Ni}$. More specifically, the evolution of the proton single-particle energies will be discussed as a function of neutron number, together with the consequences for the occupation of intruder configurations and the onset of deformation and collectivity.

\section{Evolution of the $Z=28$ shell gap}

Changes in the single-particle structure are strongly related to certain properties of the (residual) interactions between nucleons. In particular the tensor component of the monopole interaction was found to be responsible for the changes in the single-particle energies (SPEs) [8]. The tensor force is attractive between two orbits with relative spin orientations $j_{>}=l+1 / 2$ and $j_{<}^{\prime}=l^{\prime}-1 / 2$ (or $j_{<}=l-1 / 2$ and $\left.j_{>}^{\prime}=l^{\prime}+1 / 2\right)$ and repulsive between those with spin orientations $j_{>}$and $j_{>}^{\prime}\left(\right.$ or $j_{<}$and $\left.j_{<}^{\prime}\right)$.

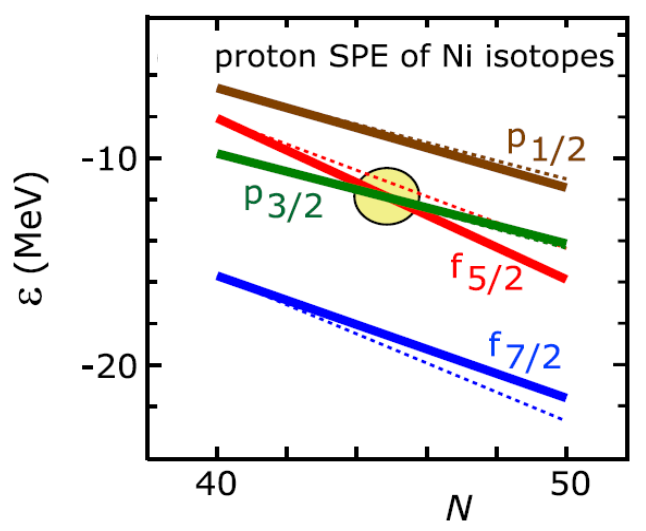

Figure 2. Calculated proton SPEs for the Ni chain. Figure is taken from Ref. [9]. The same color code is also used in Figures 3,4 , and 6 .

The evolution of the $Z=28$ shell gap was previously investigated by systematically studying the properties of the $\mathrm{Ni}$ isotopes from ${ }^{68} \mathrm{Ni}(N=40)$ to ${ }^{78} \mathrm{Ni}$ $(N=50)$. Figure 2 shows the resulting proton SPEs as a function of neutron number from shell model calculations for the Ni isotopes [9]. Note that the SPEs change more dramatically when including the tensor component of the monopole interaction (solid lines), while the relative changes are less pronounced when the tensor component is not included (dashed lines). According to these calculations, the changes in the proton SPEs lead to an inversion of the $\pi 2 p_{3 / 2}$ and $\pi 1 f_{5 / 2}$ orbitals with increasing number of neutrons in the $v 1 g_{9 / 2}$ orbital. The crossing of the $\pi 2 p_{3 / 2}$ and $\pi 1 f_{5 / 2}$ orbitals is consistent with the measured $5 / 2^{-}$ spin-parity for the ground state in ${ }^{75} \mathrm{Cu}$ [10]. Furthermore, the calculations predict a reduction for the $Z=28$ shell gap as a result of the attraction between the $\pi 1 f_{5 / 2}$ and the $v 1 g_{9 / 2}$ and the repulsion between the $\pi 1 f_{7 / 2}$ and $v 1 g_{9 / 2}$ orbitals.

Proton SPEs as a function of neutron number can be determined by studying single-proton excitations in the $\mathrm{Cu}$ $(Z=29)$ isotopes, where the last uncoupled proton resides above the $Z=28$ gap in the $f_{5 / 2} p$ shell $\left(1 f_{5 / 2}, 2 p_{3 / 2}\right.$, $\left.2 p_{1 / 2}\right)$. Indeed, the neutron-rich copper isotopes from ${ }^{69} \mathrm{Cu}$ $(\mathrm{N}=40)$ to ${ }^{79} \mathrm{Cu}(\mathrm{N}=50)$ have played an important role to study the single-particle structure in the ${ }^{78} \mathrm{Ni}$ region. Previous experimental and theoretical studies, however, indicated that the low-lying states in $\mathrm{Cu}$ nuclei can have different origins $[11,12]$ and can be classified into the following types:

- the $3 / 2^{-}$and $5 / 2^{-}$"single-particle" states, dominated by the single-proton configurations, $\pi 2 p_{3 / 2}$ and $\pi 1 f_{5 / 2}$, respectively,

- the "particle-core coupled" states from $9 / 2^{-}$to $1 / 2^{-}$and from $7 / 2^{-}$to $1 / 2^{-}$will appear as a result of the $\pi 2 p_{3 / 2}$ and $\pi 1 f_{5 / 2}$ single-particle excitations coupled to the first excited $2^{+}$state of the even-even $\mathrm{Ni}$ core, denoted as $2 p_{3 / 2} \otimes 2_{1}^{+}$and $1 f_{5 / 2} \otimes 2_{1}^{+}$, respectively

- the 7/2- "intruder" state arises from the excitation of a proton from the $1 f_{7 / 2}$ orbital across the $Z=28$ shell gap, leaving a hole in the $1 f_{7 / 2}$ orbit, i.e. $\pi 1 \mathrm{f}_{7 / 2}^{-1}$.

\subsection{Beta-decay of ${ }^{75,77} \mathrm{Ni}$}

$\mathrm{Cu}$ nuclei have been studied in detail up to ${ }^{73} \mathrm{Cu}$ via different reaction mechanisms such as $\beta$-decay, Coulomb excitation, and single- and multi-nucleon transfer reaction studies [13-21]. For the heavier isotopes ${ }^{75} \mathrm{Cu}$ and ${ }^{77} \mathrm{Cu}$, only the spin and parity of the ground state was known [10], and, in case of ${ }^{75} \mathrm{Cu}$, two low-lying isomeric states [22, 23]. To identify low-lying states and establish the level schemes for ${ }^{75} \mathrm{Cu}$ and ${ }^{77} \mathrm{Cu}, \beta$-decay experiments were carried out at RI Beam Factory (RIBF) of the RIKEN Nishina Center [24]. The secondary beam particles near ${ }^{78} \mathrm{Ni}$ were produced via fission reaction of the ${ }^{238} \mathrm{U}$ primary beam on a thick ${ }^{9} \mathrm{Be}$ target. After particle identification in atomic number $(Z)$ and mass-to-charge ratio $(A / Q)$ using the $T O F-B \rho-\Delta E$ technique [25] in the BigRIPS fragment separator $[26,27]$, the ions were delivered to the experimental setup through the ZeroDegree Spectrometer (ZDS) [27] and implanted in a stack of eight highly pixelised double-sided silicon strip detectors (WAS3ABI [28]). Each DSSSD had 60 horizontal and 40 vertical strips of $1 \mathrm{~mm}$ pitch, respectively. Surrounding the implantation detector was the EURICA germanium detector array $[28,29]$ for the detection of $\gamma$ rays emitted after $\beta$ decay of the implanted ions. 


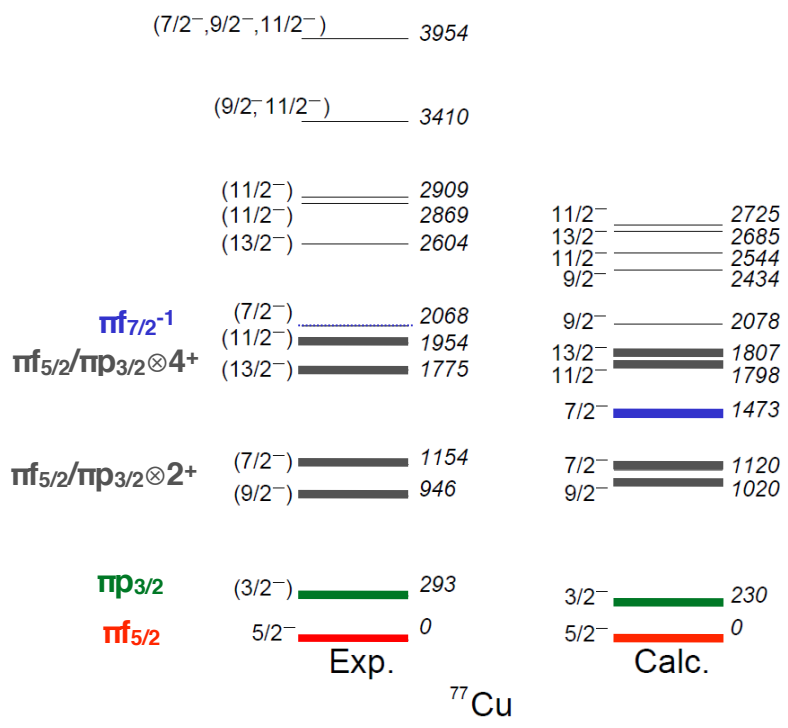

Figure 3. Excited states of ${ }^{77} \mathrm{Cu}$ compared to the MCSM calculations. The orbital configurations are indicated for the lowest states.

The level structure of ${ }^{75,77} \mathrm{Cu}$ was investigated through $\gamma$-ray spectroscopy following the $\beta$ decay of ${ }^{75,77} \mathrm{Ni}$. The implanted $\mathrm{Ni}$ ions were correlated on an event-by-event basis in time and position with $\beta$ decays detected in WAS3ABI. It was required that the $\beta$-decay electrons were detected in the same DSSSD within a correlation area that covered up to two pixels away from the implantation position. The time correlation between $\beta$-decay events and detected $\gamma$ rays was used to identify $\gamma$-ray transitions as originating from ${ }^{75,77} \mathrm{Cu}$ or their respective daughter decays. Due to the high efficiency of EURICA it was possible to analyze $\gamma-\gamma$ coincidences and use the coincidence relationships to build level schemes up to around $4 \mathrm{MeV}$ excitation energy for both nuclei. A total of 28 new excited states were identified in ${ }^{75} \mathrm{Cu}$ [33] and 12 in ${ }^{77} \mathrm{Cu}$ [32]. Tentative spin-parity values were assigned for all states in ${ }^{77} \mathrm{Cu}$ based on the $\beta$-decay feeding $(\log (f t)$ values $)$ and $\gamma$ decay properties, whereas this was only possible for the lowest states in ${ }^{75} \mathrm{Cu}$. Figure 3 shows the level scheme for ${ }^{77} \mathrm{Cu}$ obtained from the present data together with results from state-of-the-art Monte Carlo Shell Model calculations [30, 31]. The calculations used the A3DA interaction [30] with rather large valence space, i.e. the full $f p$ shell $\left(1 f_{7 / 2}, 1 f_{5 / 2}, 2 p_{3 / 2}, 2 p_{1 / 2}\right)$ plus the $1 g_{9 / 2}$ and $2 d_{5 / 2}$ orbitals outside a ${ }^{40} \mathrm{Ca}$ core.

There is rather good agreement between the experimental results and the results from the shell model calculations, in particular for the states up to $2 \mathrm{MeV}$ excitation energy. All low-lying states are explained as having either single-particle, core-coupled, or intruder character as explained above. With new experimental results available, the shell model calculations have been refined compared to the previous results shown in Figure 2. The SPEs from the new shell model calculations are shown in Figure 4. The results indicate that the inversion of the $\pi 2 p_{3 / 2}$ and $\pi 1 f_{5 / 2}$ orbitals does not occur at $N=46\left({ }^{75} \mathrm{Cu}\right)$, as was

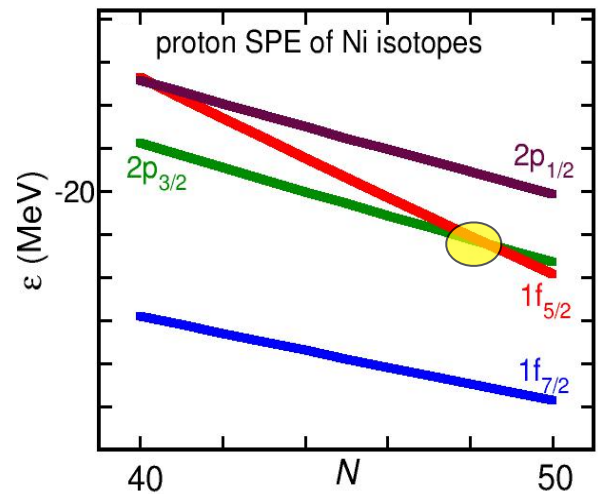

Figure 4. Calculated proton SPEs for the Ni chain. Figure is adapted from Ref. [32].

reported in 2010 in the work of Otsuka et al. [9], but occurs instead at $N=48\left({ }^{77} \mathrm{Cu}\right)$. The fact that the $5 / 2^{-}$state is lower than the $3 / 2^{-}$state already in ${ }^{75} \mathrm{Cu}$ is explained by correlation effects due to multipole interaction, which are stronger for the $5 / 2^{-}$state than for the $3 / 2^{-}$state [32]. Furthermore, with the crossing of these two orbits found to occur later than previously thought, the reduction of the $Z=28$ shell gap is also smaller than in previously calculations. As one of the main results from the present $\beta$ decay study it can be concluded that the size of the $Z=28$ shell gap is reduced from $6.5 \mathrm{MeV}$ at $N=40$ to $5 \mathrm{MeV}$ at $N=50$ [32]. More detailed discussions, together with extended results on ${ }^{75} \mathrm{Cu}$ and their comparison to the largescale shell-model calculations, will be presented in a forthcoming article [33].

\section{Proton excitations across $Z=28$}

The $\beta$-decay experiment discussed above gave insight into the single-particle properties in the ${ }^{78} \mathrm{Ni}$ region based on comparisons with shell model calculations. It also gave a first indication for a $7 / 2^{-}$intruder state based on a proton excitation across the $Z=28$ shell gap (see Figure 4). Complementary experiments using direct reaction mechanism such as one-proton knockout selectively populate excited states arising from single-proton excitations. Such experiments can give a more direct identification of singleparticle states based on $2 p_{3 / 2}$ and $1 f_{5 / 2}$ protons, and, in particular, of the $\left(\pi 1 f_{7 / 2}\right)^{-1}$ particle-hole excitation across the $Z=28$ shell gap. As the interpretation of the $7 / 2^{-}$ states found in the $\beta$-decay experiment is less straightforward as compared to the other low-lying states, complementary knock-out experiments can provide essential information on the evolution of the shell structure close to ${ }^{78} \mathrm{Ni}$. The location of the $7 / 2^{-} 2 p-1 h$ intruder state along the chain of the $\mathrm{Cu}$ isotopes is important to provide sensitive information on the expected shape coexistence and onset of deformation in the ${ }^{78} \mathrm{Ni}$ region [31].

\subsection{One-proton removal reaction}

Experiments to study neutron-rich $\mathrm{Cu}$ isotopes in knockout reactions were performed within the SEASTAR col- 
laboration and campaign at RIKEN. The ${ }^{75,77} \mathrm{Cu}$ nuclei were produced through one-proton knockout of ${ }^{76,78} \mathrm{Zn}$ beam particles. In terms of reaction mechanism, the oneproton knockout strength is expected to be largest for the $1 \mathrm{f}_{7 / 2}$ orbital since there are almost 8 protons in the groundstate configuration of the ${ }^{76,78} \mathrm{Zn}$ isotopes, while the remaining single-particle strength due to 2 protons above $Z=28$ will be mostly shared between the $1 \mathrm{f}_{5 / 2}, 2 \mathrm{p}_{3 / 2}$, and $1 \mathrm{p}_{1 / 2}$ orbitals. As a consequence, the cross section to populate the $7 / 2^{-}$particle-hole state is expected to be large, and the corresponding state can be clearly identified.

The experiment used again an intense primary beam of ${ }^{238} \mathrm{U}$ and a primary ${ }^{9} \mathrm{Be}$ target. The BigRIPS separator was optimized to select neutron-rich $\mathrm{Zn}$ isotopes. Identification of the incoming projectiles and outgoing reaction products was performed on event-by-event basis similar to the technique mentioned in Sect. 2.1. For the purpose of one-proton knockout, a reaction target of $10 \mathrm{~cm}$ thick liquid hydrogen, surrounded by the active target time projection chamber MINOS [34], was exploited during the experiment. The DALI2 array [35], coupled to the MINOS device, was used for the detection of the $\gamma$ rays emitted from the reaction products. The setup allowed performing event-by-event Doppler correction of the $\gamma$ rays detected in DALI2 using the reaction vertex information reconstructed in MINOS. Details of the procedure can be found in Ref. [36].

Figure 5 shows the Doppler-corrected $\gamma$-ray spectra obtained for ${ }^{75} \mathrm{Cu}$ and ${ }^{77} \mathrm{Cu}$ resulting from the ${ }^{76} \mathrm{Zn}(p, 2 p){ }^{75} \mathrm{Cu}$ and ${ }^{78} \mathrm{Zn}(p, 2 p){ }^{77} \mathrm{Cu}$ knockout reactions ,respectively. Eight transitions of 490(10), 530(10), 880(10), 950(10), 990(10) 1410(20), 1480(20), and $1680(30) \mathrm{keV}$ energies are visible in the spectrum for ${ }^{75} \mathrm{Cu}$ (Figure 5a) while six transitions of 290 (10), 860(20), 950(20), 1150(20), 1740(30), and 2060(20) keV are seen in in the spectrum for ${ }^{77} \mathrm{Cu}$ (Figure $5 \mathrm{~b}$ ). The knowledge of the level schemes of ${ }^{75} \mathrm{Cu}$ and ${ }^{77} \mathrm{Cu}$ from the $\beta$-decay experiments was of great help for the identification of transitions in the spectra following the knock-out reactions. In fact, all $\gamma$-ray transitions indicated in Figure 5 were also seen following the $\beta$ decay of ${ }^{75} \mathrm{Ni}$ and ${ }^{77} \mathrm{Ni}$, respectively [32, 33].

The transition at $290 \mathrm{keV}$ lies in the low-energy region where a large atomic background makes its identification difficult. The spectrum shown in the inset of Figure 5b was obtained using only DALI2 detectors at forward angles. Due to a relatively long lifetime of the $\left(3 / 2^{-}\right)$state at $290 \mathrm{keV}$ excitation energy, its decay transition appears shifted to $260 \mathrm{keV}$ under these conditions. Using the lineshape of this transition depopulating the level at $290 \mathrm{keV}$, a lower limit of about $0.5 \mathrm{~ns}$ for the half life of the $\left(3 / 2^{-}\right)$ state could be extracted in the present experiment. Figure 6 shows examples of the coincidence spectra gated on the 530- and $490-\mathrm{keV}$ transitions in ${ }^{75} \mathrm{Cu}$. The coincidence relation between $530 \mathrm{keV}$ and $880 \mathrm{keV}$ and $950 \mathrm{keV}$ and between $490 \mathrm{keV}$ and $990 \mathrm{keV} \gamma$-ray transitions confirms the level scheme obtained in Ref. [33]. The similar analysis has been performed for ${ }^{77} \mathrm{Cu}$ and confirms the level scheme reported in Ref. [32]

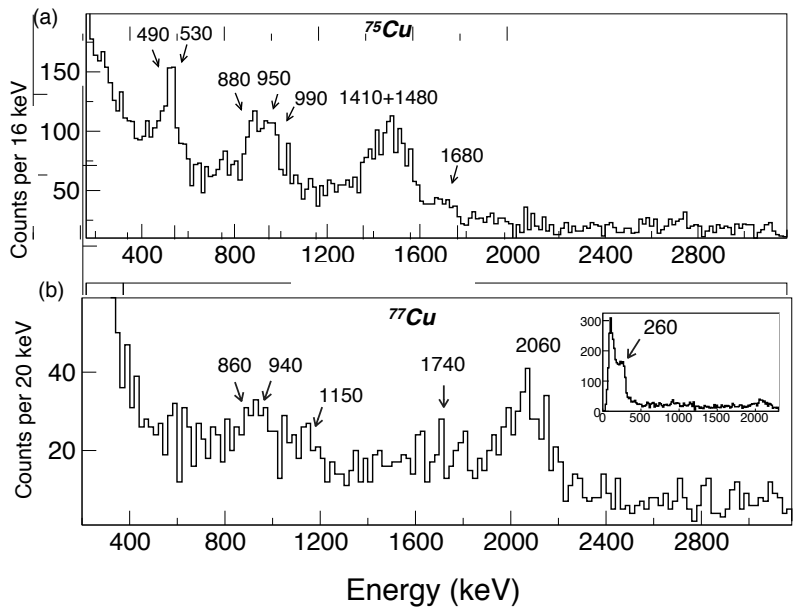

Figure 5. Doppler corrected $\gamma$-ray spectra (a) for ${ }^{75} \mathrm{Cu}$ and (b) for ${ }^{77} \mathrm{Cu}$. Inset: The same spectrum as (b) obtained only using DALI2 detectors at forward angles.

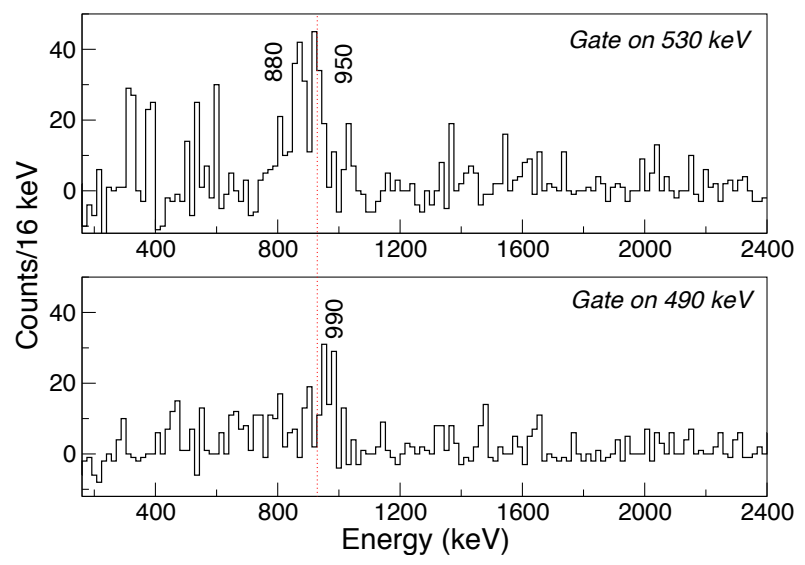

Figure 6. Background subtracted $\gamma-\gamma$ coincidences for ${ }^{75} \mathrm{Cu}$. The spectra gated on $530-\mathrm{keV}$ and on $490-\mathrm{keV}$ transitions are shown in upper and bottom panel, respectively.

From Figure 5 it is evident that the transitions at around $1.5 \mathrm{MeV}$ in ${ }^{75} \mathrm{Cu}$ and $2 \mathrm{MeV}$ in ${ }^{77} \mathrm{Cu}$ are more strongly populated compared to the other transitions seen in the spectra. Accordingly, the state at $1483 \mathrm{keV}$ in ${ }^{75} \mathrm{Cu}$ and at $2068 \mathrm{keV}$ in ${ }^{77} \mathrm{Cu}$ will have the largest population through the $(\mathrm{p}, 2 \mathrm{p})$ reaction and, hence, can be assigned as the states based on the $\left(\pi 1 f_{7 / 2}\right)^{-1}$ particle-hole excitation. The detailed analysis including extraction of the exclusive cross sections and spectroscopic factors will be reported in another forthcoming article [37].

The preliminarily results from the one-proton knockout and beta-decay experiments at RIKEN can be translated into systematics of the excited states along the $\mathrm{Cu}$ chain. Figure 7 shows the energy of the low-lying states of the $\mathrm{Cu}$ isotopes as a function of the neutron number. From the systematics we can make the following observations:

- An increase in the energy of the $3 / 2^{-}$single-particle state (green full dots) and a decrease in the $5 / 2^{-}$singleparticle state (red full squares) state is clearly visible as a result of the monopole tensor interaction, which is re- 
pulsive between the $\pi 2 p_{3 / 2}$ and $v 1 g_{9 / 2}$ and strongly attractive between the $\pi 1 f_{5 / 2}$ and $v 1 g_{9 / 2}$ orbitals, respectively.

- The particle-core coupled states (orange full diamonds) dominate the higher-energy regions. For the lighter isotopes from $N=36$ to 44 one observes $7 / 2^{-}$and $11 / 2^{-}$ states based largely on the $2 p_{3 / 2} \otimes 2_{1}^{+}$coupling, while for the heavier isotopes from $N=46$ to 48 one observes $9 / 2^{-}$and $13 / 2^{-}$states based largely on the $1 f_{5 / 2} \otimes 2_{1}^{+}$coupling. All these core-coupled states follow very closely the trend of the $2_{1}^{+}$and $4_{1}^{+}$states in the corresponding ${ }^{(A-1)} \mathrm{Ni}$ core.

- The 7/2- "intruder" state (blue full triangle) exhibits a parabolic behavior indicating a possible existence of shape coexistence along the copper chain from $N=40$ to $N=50$. Similar parabolic behaviors have been observed in different mass regions such as the $\mathrm{Hg}(\mathrm{Z}=80)$, $\mathrm{Pb}(\mathrm{Z}=82)$, and $\mathrm{Sn}(\mathrm{Z}=50)$ isotopic chains $[38,39]$.

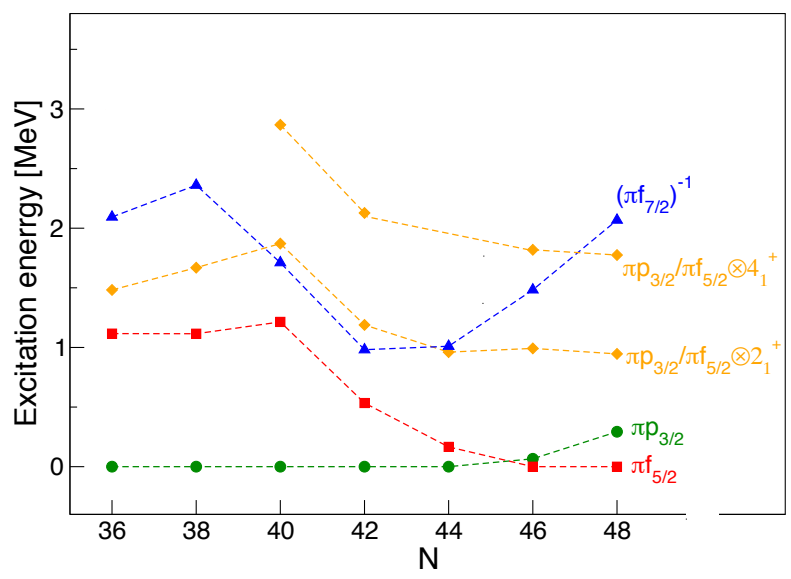

Figure 7. Systematics of the low-lying states in the $\mathrm{Cu}$ nuclei. Experimental values are from References [7, 12, 14, 32, 33]

\section{Conclusion}

In conclusion, the status of the current spectroscopic studies for ${ }^{75} \mathrm{Cu}$ and ${ }^{77} \mathrm{Cu}$ performed at RIKEN has been discussed. Level schemes up to around $4 \mathrm{MeV}$ were constructed for both nuclei from the $\beta$-decay studies of ${ }^{75} \mathrm{Ni}$ and ${ }^{77} \mathrm{Ni}$ within the EURICA campaign. The singleparticle character of the low-lying states was identified more selectively via one-proton knockout reactions of ${ }^{76} \mathrm{Zn}$ and ${ }^{78} \mathrm{Zn}$ projectiles on a proton target within the SEASTAR campaign. The results were compared to largescale shell model calculations, yielding information about the single-particle structure in the ${ }^{78} \mathrm{Ni}$ region in general and the size of the $Z=28$ shell gap in particular. The results allow a significant extension of the systematics along the chain of $\mathrm{Cu}$ isotopes. The identification of intruder states suggest that deformation and shape coexistence are important for the ${ }^{78} \mathrm{Ni}$ region. Further results and more detailed discussions will be presented in forthcoming publications.

\section{References}

[1] B. Bastin et al., Phys. Rev. Lett. 99 (2007) 022503.

[2] T. Glasmacher et al., Phys. Lett. B395 (1997) 163.

[3] H.Scheit et al., Phys.Rev.Lett. 77, 3967 (1996)

[4] H.N. Liu et al., Phys.Rev.Lett. 122, 072502 (2019).

[5] C. Force et al., Phys.Rev.Lett. 105, 102501 (2010).

[6] R. Taniuchi et al., Nature (London) 569, 53 (2019).

[7] NNDC-National Nuclear Data Center, https://www.nndc.bnl.gov.

[8] T. Otsuka, T. Suzuki, R. Fujimoto, H. Grawe, and Y. Akaishi, Phys. Rev. Lett. 95, 232502 (2005).

[9] T. Otsuka et al., Phys. Rev. Lett. 104, 012501 (2010).

[10] K.T. Flanagan et al., Phys. Rev. Lett. 103, 142501 (2009).

[11] A.M. Oros-Peusquens and P.F. Mantica., Nucl. Phys. A 669 (2000).

[12] T. Ishii, M. Asai,M. Makishima, I. Hossain, M. Ogawa, J. Hasegawa, M. Matsuda, and S. Ichikawa, Phys. Rev. Lett. 84, 39 (2000).

[13] S. Franchoo et al., Phys. Rev. Lett. 81, 3100 (1998).

[14] S. Franchoo et al., Phys. Rev. C 64054308 (2001).

[15] I. Stefanescu et al., Phys. Rev. Lett. 100, 112502 (2008).

[16] I. Stefanescu et al., Phys. Rev. Lett. 100, 112502 (2008).

[17] B. Zeidman and J. A. Nolen, Phys. Rev. C18, 2122 (1978).

[18] P. Morfouace et al., Phys.Lett. B 751, 306 (2015).

[19] P. Morfouace et al., Phys.Rev. C 93, 064308 (2016).

[20] M. Doncel et al., Acta Phys. Pol. B44, 505 (2013).

[21] E. Sahin et al., Phys. Rev. C 91034302 (2015).

[22] J.M. Daugas et al., Phys. Rev. C 81, 034304 (2010).

[23] C. Petrone at al., Phys. Rev. C 94, 024319 (2016).

[24] T. Onishi et al., J. Phys. Soc. Jpn. 79, 073201 (2010).

[25] N. Fukuda, T. Kubo, T. Ohnishi, N. Inabe, H. Takeda, D. Kameda, and H. Suzuki, Nucl. Instrum. Methods Phys. Res., Sect. B 317323 (2013).

[26] T. Kubo, Nucl. Instrum. Methods Phys. Res., Sect. B 20497 (2003).

[27] T. Kubo et al., Prog. Theor. Exp. Phys. 2012 3C003 (2012).

[28] S. Nishimura, Prog. Theor. Exp. Phys. $201203 \mathrm{C} 006$ (2012).

[29] P.-A. Söderström, S. Nishimura, P. Doornenbal, G. Lorusso, T. Sumikama, H. Watanabe, Z. Xu, H. Baba, F. Browne, S. Go et al., Nucl. Instrum. Methods Phys. Res., Sect. B 317649 (2013).

[30] N. Shimizu, T. Abe, Y. Tsunoda, Y. Utsuno, T. Yoshida, T. Mizusaki, M. Honma, and T. Otsuka, Prog. Theor. Exp. Phys. 2012 01A205 (2012).

[31] Y. Tsunoda, T. Otsuka, N. Shimizu, M. Honma, and Y. Utsuno, Phys. Rev. C 89, 031301(R) (2014).

[32] E. Sahin et al., Phys. Rev. Lett. 118, 242502 (2017).

[33] F. Bello Garrote et al., submitted to Phys. Rev. C.

[34] A. Obertelli et al., Eur. Phys. J. A 50, 8 (2014). 
[35] S. Takeuchi, T. Motobayashi, Y. Togano, M. Matsushita, N. Aoi, K. Demichi, H. Hasegawa, and H. Murakami, Nucl. Instrum. Methods Phys. Res., Sect. A 763, 596 (2014).

[36] C. Santamaria et al., Phys. Rev. Lett. 115, 192501 (2015).
[37] E. Sahin et al., submitted to Phys. Rev. Lett.

[38] J. Elseviers et al., Phys. Rev. C 84034307 (2011).

[39] K. Heyde and J. L. Wood, Rev. Mod. Phys. 83, 1467 (2011). 